Otol Neurotol. 2018 September ; 39(8): 1005-1011. doi:10.1097/MAO.0000000000001929.

\title{
Acute Otitis Media and Associated Complications in United States Emergency Departments
}

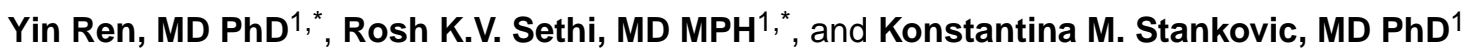
${ }^{1}$ Department of Otolaryngology, Massachusetts Eye and Ear, Harvard Medical School, Boston, Massachusetts, USA

\section{Abstract}

Objective-Complications due to acute otitis media (AOM), while rare, are associated with significant morbidity and are not well characterized from an epidemiological perspective. We analyze the pattern of presentation and emergency department (ED) utilization in patients with $\mathrm{AOM}$ and associated complications.

Study Design-Retrospective analysis of the Nationwide Emergency Department Sample (NEDS) from 2009 to 2011.

Setting-Emergency Department.

Patients-Patients who presented with a primary diagnosis of AOM or acute mastoiditis.

Intervention-Diagnostic.

Main outcome measures-NEDS was queried for patient encounters with a diagnosis of AOM or acute mastoiditis based on ICD-9 codes. Complications of severe infection, including petrositis, Gradenigo's syndrome, facial paresis, labyrinthitis, meningitis, intracranial abscess, venous sinus thrombosis, and cerebrospinal fluid leak were assessed. Weighted estimates for demographics, types of complications, socioeconomic status, and trends over time were extracted.

Results-A weighted total of 5,811,127 ED visits were identified. The majority of patients were less than 18 years old (79.9\%) with an average age of 10.1 years. Most were discharged (99.4\%). There were 15,243 $(0.26 \%)$ patients who presented with a complication. The most common complications were acute mastoiditis $(0.16 \%)$, labyrinthitis $(0.06 \%)$ and facial paresis $(0.03 \%)$. Compared to patients with uncomplicated AOM, patients with complicated AOM were older (37 vs. 10 years old), insured by Medicare (18\% vs. $2.1 \%$ ), and more likely to be admitted (43.6\% vs. $0.4 \%)$ ( $p<0.0001$, for all comparisons).

Conclusions-ED visits related to AOM or mastoiditis are common and complications are rare. An in-depth analysis on a national level is useful for assessing healthcare utilization trends.

CORRESPONDING AUTHOR: Yin Ren, MD, PhD, Department of Otolaryngology, Massachusetts Eye and Ear, Harvard Medical School, 243 Charles Street, Boston, MA 02114, USA, yin_ren@meei.harvard.edu.

Equal contributions

Presentation: This was presented as an oral presentation at the $151^{\text {st }}$ Annual Spring Meeting of the American Otologic Society, National Harbor MD, April $21^{\mathrm{st}}, 2018$

FINANCIAL DISCLOSURE: The authors have indicated they have no financial relationships relevant to this article to disclose. POTENTIAL CONFLICT OF INTEREST: The authors have indicated they have no potential conflicts of interest to disclose. 


\section{Keywords}

Acute otitis media; mastoiditis; complications; emergency department; NEDS; resource utilization

\section{INTRODUCTION}

Acute otitis media (AOM) is one of the most common childhood infections that affects approximately $50 \%$ to $85 \%$ of children before the age of three, and remains the leading cause of visits to pediatricians (1). It accounts for over 20 million clinic visits and two to three million emergency department (ED) visits annually (2). AOM presents a significant health care utilization burden with an estimated cost of nearly $\$ 1$ billion annually $(3,4)$. Children with AOM manifest an increase in outpatient health care costs of $\$ 314$, two more office visits, and 0.2 more ED visits per child annually compared to those without AOM (5). In the adult population, AOM can be associated with serious intracranial infections such as bacterial meningitis (6). With a growing shortage of primary care providers, AOM remains one of the most common diagnoses encountered in EDs in the United States.

In the pre-antibiotic era, infectious complications occurred in up to six percent of patients with AOM or acute mastoiditis, many of which were fatal (7). Serious complications include sigmoid sinus thrombosis, epidural or intracerebral abscess, meningitis, petrous apicitis, and otic hydrocephalus. Since the introduction of antibiotics and the pneumococcal vaccination, the incidence of suppurative complications has declined to under one percent (8). Nonetheless, otogenic intracranial complications can be associated with significant morbidity and dire sequela if not treated promptly, owing to the proximity of the middle ear to the skull base (9) and the non-specificity of presenting symptoms (10). Therefore, a thorough understanding of the clinical factors associated with complications from AOM is crucial to facilitate swift diagnosis, timely triage, and involvement of appropriate subspecialists.

Accordingly, we aim to characterize the incidence and prevalence of AOM and associated complications in both children and adults, using the largest publically available national ED database. We also provide in-depth analysis of patient demographics, geographic and temporal trends, and procedures performed in the ED. Finally, we utilize multivariate regression analysis to identify clinical factors that are associated with the development of intratemporal and intracranial complications from AOM.

\section{MATERIALS AND METHODS}

This study was exempt from Massachusetts Eye and Ear Infirmary Institutional Review Board approval. The 2009 through 2011 Nationwide Emergency Department Sample (NEDS), published by the Healthcare Cost and Utilization Project (HCUP) and sponsored by the Agency for Healthcare Research and Quality, was used to estimate the national prevalence of acute otitis media and acute mastoiditis, and complications therein. NEDS is the largest publically available, all-payer ED database in the United States. It contains discharge abstracts for $86,866,759 \mathrm{ED}$ visits at 951 hospitals in 30 states and provides a $20 \%$ stratified sample of all hospital-based EDs in the country between 2009 and 2011. Individual 
ED encounters can be weighted according to the sample design, therefore the 2009 through 2011 NEDS provides a national weighted sample of over 388 million ED visits. In the NEDS, all diagnostic and therapeutic procedures are recorded using International Classification of Diseases, Ninth Revision, Clinical Modification (ICD-9-CM) procedure codes.

All patients with a diagnosis of acute otitis media (AOM) with or without acute mastoiditis were included in the study (ICD-9CM 381.x, 382.x, 383.x). Descriptive analysis was performed to characterize patient demographic variables including age, gender, and national quartile for median household income (categorized by HCUP according to patient zip code). Additional variables analyzed include insurance payer, visit characteristics (day of the week and month), and characteristic of the hospital (teaching status, trauma designation, and geographic region).

Complications, including acute mastoiditis, labyrinthine fistula, facial paresis, subperiosteal abscess, Gradenigo syndrome, meningitis, venous sinus thrombosis, petrositis, intracranial abscess, cerebrospinal fluid otorrhea, and labyrinthine fistula were tabulated. The ICD-9CM codes are listed in Supplementary Table 1. NEDS is a stratified two-stage cluster sample; therefore, discharge weights and standard formulas for a two-stage cluster sample were used to generate national estimates and calculate standard errors (SEs). Patient demographics, visit, injury, and hospital characteristics were compared by patient disposition (admission vs. treatment and release) in bivariable analysis. Differences in proportions and means were assessed using sample design adjusted statistical tests including the Wald chi-square test (for proportions) and two-sided unpaired $\mathrm{t}$ tests (for means).

Significant predictors of complicated otitis media were determined in a multivariable logistic regression. Complex survey sample design was accounted for in the regression analysis. Each model was inclusive of all independent variables previously listed (patient demographics, visit characteristics, hospital characteristics) to account for all potential predictors available in NEDS. Statistical significance was determined by a type I error threshold of .05. Data linkages and statistical analysis were performed using SAS version 9.3 (SAS Institute, Cary, NC).

\section{RESULTS}

\section{Emergency Department Visits for Acute Otitis Media}

There was a weighted total of 388,904,009 ED visits from 2009 through 2011. A total of $5,811,127$ visits were attributed to a primary diagnosis of acute otitis media (AOM) or acute mastoiditis, representing $1.49 \%$ of all ED visits. The annual volume of otitis media-related visits remained relatively stable between 2009 and 2011: there were 2,030,340 visits in 2009, 1,952,485 visits in 2010, and 1,928,301 visits in 2011.

\section{Common Complications from AOM}

The 12 most common complications associated with AOM in patients who presented to the ED are listed in Table 1. These included both intratemporal (extracranial) and intracranial complications. The most common extracranial complications amongst all age groups were 
acute mastoiditis $(\mathrm{N}=9,387,0.16 \%)$, labyrinthitis $(\mathrm{N}=3,402,0.06 \%)$, facial paresis $(\mathrm{N}=$ $1,677,0.03 \%)$, and subperiosteal abscess $(\mathrm{N}=560,0.01 \%)$.

\section{Patient Demographics Associated with AOM Complications}

A weighted total of 15,243 visits with a primary complaint of AOM were associated with any type of complication, representing $0.26 \%$ of all visits. Demographic statistics are outlined in Table 2. Amongst all patients who presented a primary diagnosis of AOM or acute mastoiditis, the average age was 10.1 years old (standard error of the mean [SEM] = $0.1)$ and $79.9 \%$ of patients were children under 18 years of age $(\mathrm{N}=4,643,868)$. There was no gender predominance. More patients visited the ED during the weekdays than the weekend $(65.7 \%)$, were from low income $(<\$ 39,000,35.4 \%)$ or medium income households $(<\$ 48,000,30.9 \%)$, and were insured by Medicaid $(54.7 \%)$ or private insurance $(27.5 \%)$. Only a small fraction of patients was evaluated at a designated trauma hospital $(24.2 \%)$.

Bivariable comparisons for demographic factors for patients who presented with complicated AOM or acute mastoiditis versus patients with uncomplicated AOM are presented in Table 2. The average age of patients who presented to the ED with complicated AOM was 37.0 years $(\mathrm{SEM}=0.6)$. Compared to patients with uncomplicated AOM, patients with complicated AOM were more likely to be adults greater than 18 years of age $(75.9 \%$ vs. $19.9 \%, P<0.0001)$, female ( $55.4 \%$ vs. $49.9 \%, P<0.0001)$, from higher income households ( $>\$ 64,000,16.5 \%$ vs. $12.0 \%, P<0.0001)$, and present during the week $(70.3 \%$ vs. $65.7 \%, P<0.0001)$. Compared to patients with uncomplicated AOM, a significantly higher proportion of patients with complicated AOM were insured by Medicare (18.0\% vs. $2.1 \%, P<0.0001$ ), whereas fewer had Medicaid (29.4\% vs. $54.8 \%, P<0.0001)$.

Multivariable regression analysis is listed in Table 3. Several factors were independently predictive of complicated AOM including older age, female gender, hospital trauma designation, median household income quartile by zip code, hospital teaching status, and primary insurance.

\section{Diagnostic Tests, Interventions, and Outcomes Associated with Complications from AOM}

Most patients who presented with AOM were treated and released ( $\mathrm{N}=5,778,462 ; 99.44 \%)$ (Table 4). A total of 32,665 AOM patients were admitted, representing an overall admission rate of $0.56 \%$. Patients with complicated AOM were far more likely to be admitted than those with uncomplicated AOM (43.6\% vs. $0.4 \%, P<0.0001)$. Computed tomography (CT) of the head was performed in $0.06 \%$ ( $\mathrm{SE}=0.01$ ) of all AOM visits in the ED. The proportion of patients with AOM complications who underwent a CT scan was $2.3 \%$, compared to $0.1 \%$ of those with otherwise uncomplicated AOM $(P<0.0001)$. MRI and myringotomy with or without tube placement in the ED were infrequently performed amongst all patients; however, a greater proportion of patients with a complication re without a complication underwent MRI scanning $(0.17 \%$ vs. $0.01 \%, P=0.0311)$, and a greater proportion of patients with a complication underwent myringotomy in the ED $(0.24 \%$ vs $0.01 \%, P=0.0143)$. 


\section{Geographic and Temporal Trends in Complications Associated with AOM}

More patients present with AOM or acute mastoiditis during the winter than the summer months, with the greatest number of visits occurring between December and March, and the fewest number of visits between July and September (Figure 1). By contrast, the number of complications associated with AOM remained relatively stable throughout the year without significant variations in the month of presentation (Figure 2). The peak number of complications occurred in March $(\mathrm{N}=1,354,10.3 \%)$ and the fewest in September $(\mathrm{N}=928$, 7.0\%). Geographically, there was a significant variation in the total volume of ED visits; AOM was most common in the South ( $\mathrm{N}=2,346,494,40.4 \%)$, followed by the Midwest ( $\mathrm{N}$ $=1,506,587,25.9 \%)$, West $(\mathrm{N}=982,597,16.9 \%)$ and Northeast $(\mathrm{N}=975,449,16.8 \%)$. The geographic distribution of ED visits for complicated AOM was similar, with the South being the most predominant (37.0\%), followed by the Midwest (24.4\%), West (20.8\%), and Northeast (17.7\%) (Figure 3).

\section{DISCUSSION}

To date, this is the first study to identify presentation patterns and geographic predilections associated with complications from AOM in patients who presented to the emergency department on a national level. Numerous existing studies on otitis media complications are based on data from a single institution $(11,12)$, focused on a select set of complications $(10,13)$, and often limited to the pediatric population (14). Furthermore, a wide array of health care providers including internists, pediatricians, otolaryngologists, neurosurgeons and radiologists are involved in the care of patients with AOM, which further contributes to the fragmented nature of AOM epidemiology research. A comprehensive epidemiological analysis of complications from AOM in U.S. hospital-based EDs has not yet been performed. A better understanding of the factors associated with both extracranial and intracranial complications from AOM would be crucial for developing treatment guidelines, educating clinicians, and improving resource utilization.

Based on analysis of the NEDS data, AOM does not affect the pediatric population exclusively. While most patients were under the age of 18 , a significant proportion (20.1\%) were adults, which corresponded to about 400,000 annual visits. Strikingly, amongst patients who presented with complicated AOM, 75.9\% were adults with a mean age of 37 years. This may due to several factors. AOM typically occurs as a result of viral URI with a peak incidence between 6 and 18 months that subsequently declines with age, and most resolve without otologic or neurologic sequelae (15). Since the institution of early antimicrobial therapy for $\mathrm{AOM}$, more children are managed in the outpatient setting prior to presentation to the ED, likely reducing disease severity and rate of complications. Second, incorporation of the 7-valent pneumococcal conjugate vaccine into routine immunization schedules has led to a $17 \%$ to $28 \%$ decline in otitis media visits, which also likely reduces ED presentations from complications such as streptococcus pneumoniae meningitis (16-18). Third, the presence of co-morbid conditions such as diabetes, chronic otitis media, and cholesteatoma in adults may increase the rate of otogenic complications arising from AOM (6). Our data suggests that there is a need for heightened awareness of potential serious complications when evaluating adults with AOM in the ED. 
AOM resulted in nearly 6 million ED visits in the United States from 2009 through 2011.

Despite the large volume, nearly all patients were treated and released and only $0.56 \%$ required inpatient admission. This is consistent with other studies, where admission rate for AOM was estimated at $0.2 \%$ (19), far lower than the admission rate of $11.6 \%$ across all ED visit diagnoses amongst the pediatric population (20). The large number of ED visits related to uncomplicated AOM, a non-urgent condition typically managed in the outpatient setting, may be related to several factors. These include the young age of the mostly-pediatric patient population, greater convenience to seek ED care, difficulty in obtaining immediate access to primary care providers, and referral by the primary care physician $(21,22)$. Prior investigations on the efficacy of dedicated emergency facilities for otolaryngological complaints showed that most diagnoses were not truly emergent in nature, with the most common being impacted cerumen and otitis externa (23). However, with increased medical sub-specialization and shortage of primary care providers, non-urgent ED visits for otitis media remains common and will continue to rise $(24,25)$.

The overall rate of complications is $0.26 \%$, similar to previous studies on the natural history of AOM where rate of suppurative complications was $0.24 \%$ (8). As expected, patients with complications are more likely to be admitted than uncomplicated AOM (43.6\% vs. $0.4 \%)$. The management of mastoiditis, brain abscess and venous sinus thrombosis often requires a multidisciplinary approach in the inpatient setting. In some cases, conservative management with broad spectrum antibiotic therapy can be successful $(26,27)$. However, intracranial complications typically warrant operative intervention, which includes myringotomy and tube placement, mastoidectomy or craniotomy for abscess drainage (28). Our findings support this in that emergency-department myringotomy procedures were performed in $0.24 \%$ of patients with complications, a much higher proportion than in patients without $(0.01 \%)$. We were unable to query procedures that were performed once a patient was admitted as this was not available in NEDS.

Patients with complicated AOM are more likely to undergo CT or MRI scans in the ED. While high resolution temporal bone $\mathrm{CT}$ can be helpful in assessing mastoid bony changes and ascertaining the presence of fluid collections, the diagnosis of AOM and acute mastoiditis is largely a clinical one as incidental mastoid opacification is common (29). CT or MRI become necessary when intracranial complications are suspected, as symptoms are insidious in onset and often non-specific $(30,31)$. However, our data also suggests that a large number of CT scans were still obtained in patients with uncomplicated AOM who were discharged from the ED. Unfortunately, it is difficult to enumerate specific symptoms that may have led to obtaining the scan. Together, these findings underscore the need to further characterize the indications and utilization patterns of imaging for AOM in the emergency setting, which can add both significant cost burden and unnecessary radiation exposure.

Unsurprisingly, the number of ED visits was highest in the winter months and lowest in the summer, likely a reflection of the seasonal variation of viral respiratory illnesses closely associated with the development of AOM. The incidence of complications remained relatively constant throughout the year, which may reflect the constant presence of virulent bacterial pathogens that are more likely to cause serious complications, versus benign viral etiologies underlying respiratory tract infections which exhibit seasonal fluctuations (32). 
Geocoding analysis also revealed variations in incidence of AOM and complications in four census-defined regions of the United States. The South contained the most ED visits for both complicated and uncomplicated AOM, which may reflect underlying variations in disease burden and severity, variations in referral patterns, and differences in access to primary care providers (33).

Patients with complications are more likely to be self-pay or ensured by Medicare, whereas patients with uncomplicated AOM are more likely to have Medicaid. This is likely a reflection of the underlying demographic factors, as older patients with greater propensity for comorbidities may be independently associated with a higher likelihood of developing serious complications from AOM. Previous small-scale studies have shown that lower socioeconomic class may be associated with development of AOM in children (34-36). Patients with Medicaid may not have ready access to routine primary care, thereby increasing the likelihood of presentation to ED for otherwise uncomplicated AOM and thereby contributing to the dilutional effect. Furthermore, insured patients may have greater access to preventative care and thus only seek emergency care if symptoms suggestive of complicated AOM are present. Additional hospital-level factors including traumadesignation and hospital teaching status were predictive of visits associated with complicated AOM, likely because these centers represent tertiary referral hospitals where patients suspected of or diagnosed with complicated AOM are referred. Associations between age and complicated AOM persisted in multivariable analysis. Notably, patients from households with lower median income were at decreased odds of presenting with complicated AOM. This merits further investigation, however it may be that patients with lower socioeconomic status are not appropriately evaluated in the ED to begin with or are not recognized as having severe complications due to lack of access to preventative or primary care.

There are several limitations to the study. First, as with all large retrospective investigations utilizing national databases, the study is limited in its ability to adjust for potential unknown confounders. Second, we are unable to ascertain specific features of presentation, such as otologic exam findings, prior otologic history, microbiological data or degree of hearing loss, and correlate those with otologic outcomes including patient disposition. Third, due to limitations of the data available, we are unable to characterize the types of intervention beyond those performed in the ED. Fourth, given the lack of long-term data, we were unable to identify temporal trends over many years. Finally, we studied patients who were already diagnosed with AOM; thereby possibly missing those who presented with primarily neurologic complaints that could have arisen from an otologic process but were never formally diagnosed with otitis media.

In summary, AOM is commonly encountered in the emergency department setting. Most patients with AOM are children who do not develop suppurative extracranial or intracranial sequelae. However, one must maintain heightened awareness for such complications, particularly in the adult population with AOM. An improved understanding of the presentation patterns and clinical factors associated with complicated AOM is critical to improve hospital triage and resource utilization. 


\section{CONCLUSIONS}

We provide an in-depth epidemiological evaluation of acute otitis media in Emergency Departments in the United States and identify factors associated with development of complications from AOM. We demonstrate that although most patients with AOM are children, adults are more likely to develop complications and to be admitted. CT imaging is more frequently utilized in patients with complicated AOM. The most common suppurative complications include mastoiditis, labyrinthitis, and facial paresis. The study has implications for AOM patient triage, risk stratification, and resource utilization on a national level.

\section{Supplementary Material}

Refer to Web version on PubMed Central for supplementary material.

\section{Acknowledgments}

FUNDING SOURCE: The study was supported by NIDCD grant R01DC015824 (K.M.S.) and Nancy Sayles Day Foundation (K.M.S.).

\section{References}

1. Klein JO. The burden of otitis media. Vaccine. 2000; 19:S2-S8. [PubMed: 11163456]

2. Schappert SM. Office visits for otitis media: United States, 1975-90. Adv Data. 1992:1-19.

3. GATES G. Cost-effectiveness considerations in otitis media treatment. Otolaryngology - Head and Neck Surgery. 1996; 114:525-530. [PubMed: 8643261]

4. Kaplan B, Wandstrat TL, Cunningham JR. Overall cost in the treatment of otitis media. The Pediatric Infectious Disease Journal. 1997; 16:S9-11. [PubMed: 9041621]

5. Ahmed S, Shapiro NL, Bhattacharyya N. Incremental health care utilization and costs for acute otitis media in children. Laryngoscope. 2014; 124:301-305. [PubMed: 23649905]

6. Barry B, Delattre J, Vié F, Bedos JP, Géhanno P. Otogenic intracranial infections in adults. Laryngoscope. 1999; 109:483-487. [PubMed: 10089980]

7. Gower D, McGuirt WF. Intracranial complications of acute and chronic infectious ear disease: a problem still with us. Laryngoscope. 1983; 93:1028-1033. [PubMed: 6877010]

8. Rosenfeld RM, Kay D. Natural history of untreated otitis media. Laryngoscope. 2003; 113:16451657. [PubMed: 14520089]

9. Samuel J, Fernandes CM, Steinberg JL. Intracranial otogenic complications: a persisting problem. Laryngoscope. 1986; 96:272-278. [PubMed: 3951303]

10. Bales CB, Sobol S, Wetmore R, Elden LM. Lateral sinus thrombosis as a complication of otitis media: 10-year experience at the children's hospital of Philadelphia. PEDIATRICS. 2009; 123:709-713. [PubMed: 19171642]

11. Wanna GB, et al. Contemporary management of intracranial complications of otitis media. Otol Neurotol. 2010; 31:111-117. [PubMed: 19887978]

12. Hafidh MA, Keogh I, Walsh RMC, Walsh M, Rawluk D. Otogenic intracranial complications. a 7year retrospective review. American Journal of Otolaryngology. 2006; 27:390-395. [PubMed: 17084222]

13. Garcia RD, Baker AS, Cunningham MJ, Weber AL. Lateral sinus thrombosis associated with otitis media and mastoiditis in children. The Pediatric Infectious Disease Journal. 1995; 14:617-623. [PubMed: 7567293]

14. Leskinen K, Jero J. Complications of acute otitis media in children in southern Finland. International Journal of Pediatric Otorhinolaryngology. 2004; 68:317-324. [PubMed: 15129942] 
15. Revai K, et al. Incidence of acute otitis media and sinusitis complicating upper respiratory tract infection: the effect of age. PEDIATRICS. 2007; 119:e1408-12. [PubMed: 17545367]

16. Overturf GD, et al. Technical Report: Prevention of Pneumococcal Infections, Including the Use of Pneumococcal Conjugate and Polysaccharide Vaccines and Antibiotic Prophylaxis. PEDIATRICS. 2000; 106:367-376. [PubMed: 10920170]

17. Grijalva CG, et al. National impact of universal childhood immunization with pneumococcal conjugate vaccine on outpatient medical care visits in the United States. PEDIATRICS. 2006; 118:865-873. [PubMed: 16950975]

18. Poehling KA, et al. Reduction of frequent otitis media and pressure-equalizing tube insertions in children after introduction of pneumococcal conjugate vaccine. PEDIATRICS. 2007; 119:707715. [PubMed: 17403841]

19. Kvaerner KJ, Austeng ME, Abdelnoor M. Hospitalization for acute otitis media as a useful marker for disease severity. The Pediatric Infectious Disease Journal. 2013; 32:946-949. [PubMed: 23609040]

20. Alpern ER, et al. Epidemiology of a Pediatric Emergency Medicine Research Network. Pediatr Emerg Care. 2006; 22:689-699.

21. Uscher-Pines L, Pines J, Kellermann A, Gillen E, Mehrotra A. Emergency department visits for nonurgent conditions: systematic literature review. Am J Manag Care. 2013; 19:47-59. [PubMed: 23379744]

22. Young GP, Wagner MB, Kellermann AL, Ellis J, Bouley D. Ambulatory visits to hospital emergency departments. Patterns and reasons for use. 24 Hours in the ED Study Group. JAMA. 1996; 276:460-465. [PubMed: 8691553]

23. Sethi RKV, et al. Subspecialty emergency room as alternative model for otolaryngologic care: Implications for emergency health care delivery. American Journal of Otolaryngology. 2014; 35:758-765. [PubMed: 25106951]

24. Pitts SR, Carrier ER, Rich EC, Kellermann AL. Where Americans get acute care: increasingly, it's not at their doctor's office. Health Aff (Millwood). 2010; 29:1620-1629. [PubMed: 20820017]

25. Weinick RM, Burns RM, Mehrotra A. Many emergency department visits could be managed at urgent care centers and retail clinics. Health Aff (Millwood). 2010; 29:1630-1636. [PubMed: 20820018]

26. Tov EE, Leiberman A, Shelef I, Kaplan DM. Conservative nonsurgical treatment of a child with otogenic lateral sinus thrombosis. American Journal of Otolaryngology. 2008; 29:138-141. [PubMed: 18314028]

27. Bauer PW, Brown KR, Jones DT. Mastoid subperiosteal abscess management in children. International Journal of Pediatric Otorhinolaryngology. 2002; 63:185-188. [PubMed: 11997153]

28. Mattos JL, Colman KL, Casselbrant ML, Chi DH. Intratemporal and intracranial complications of acute otitis media in a pediatric population. International Journal of Pediatric Otorhinolaryngology. 2014; 78:2161-2164. [PubMed: 25447953]

29. Singh S, Rettiganti MR, Qin C, Kuruva M, Hegde SV. Incidental mastoid opacification in children on MRI. Pediatr Radiol. 2016; 46:704-708. [PubMed: 26914938]

30. Luntz M, Bartal K, Brodsky A, Shihada R. Acute mastoiditis: the role of imaging for identifying intracranial complications. Laryngoscope. 2012; 122:2813-2817. [PubMed: 22961223]

31. Jose J, Coatesworth AP, Anthony R, Reilly PG. Life threatening complications after partially treated mastoiditis. BMJ. 2003; 327:41-42. [PubMed: 12842957]

32. Stockmann C, et al. Seasonality of acute otitis media and the role of respiratory viral activity in children. The Pediatric Infectious Disease Journal. 2013; 32:314-319. [PubMed: 23249910]

33. Welch HG. Geographic Variation in Diagnosis Frequency and Risk of Death Among Medicare Beneficiaries. JAMA. 2011; 305:1113-1118. [PubMed: 21406648]

34. Lasisi AO, et al. Clinical and demographic risk factors associated with chronic suppurative otitis media. International Journal of Pediatric Otorhinolaryngology. 2007; 71:1549-1554. [PubMed: 17643499]

35. Vikram BK, Khaja N, Udayashankar SG, Venkatesha BK, Manjunath D. Clinico-epidemiological study of complicated and uncomplicated chronic suppurative otitis media. The Journal of laryngology and otology. 2008; 122:442-446. [PubMed: 17666139] 
36. Castagno LA, Lavinsky L. Otitis media in children: seasonal changes and socioeconomic level. International Journal of Pediatric Otorhinolaryngology. 2002; 62:129-134. [PubMed: 11788145] 


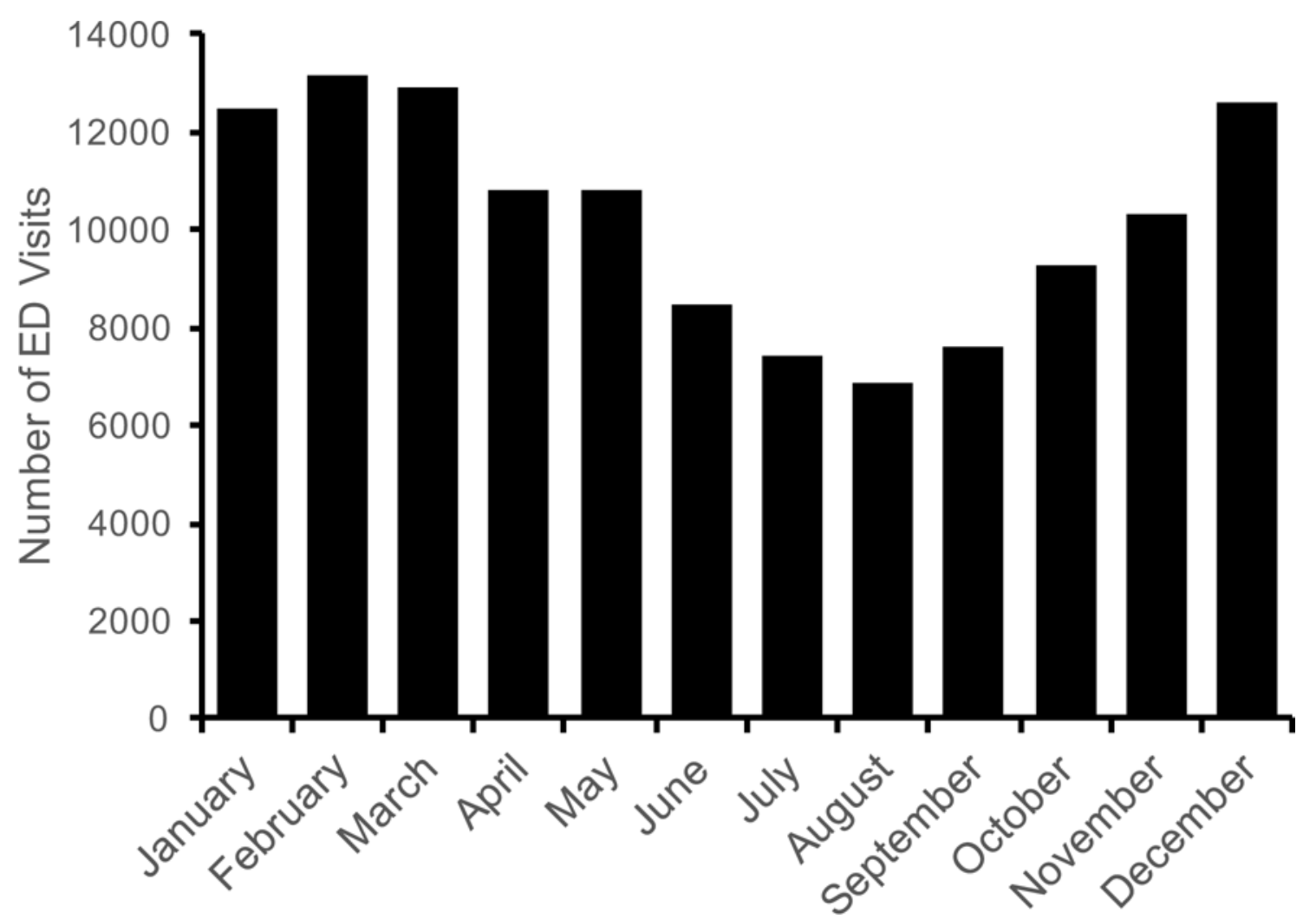

Figure 1.

Temporal trends in the volume of visits with a diagnosis of either otitis media or mastoiditis in hospital-based emergency departments in the United States (2009 through 2011). 


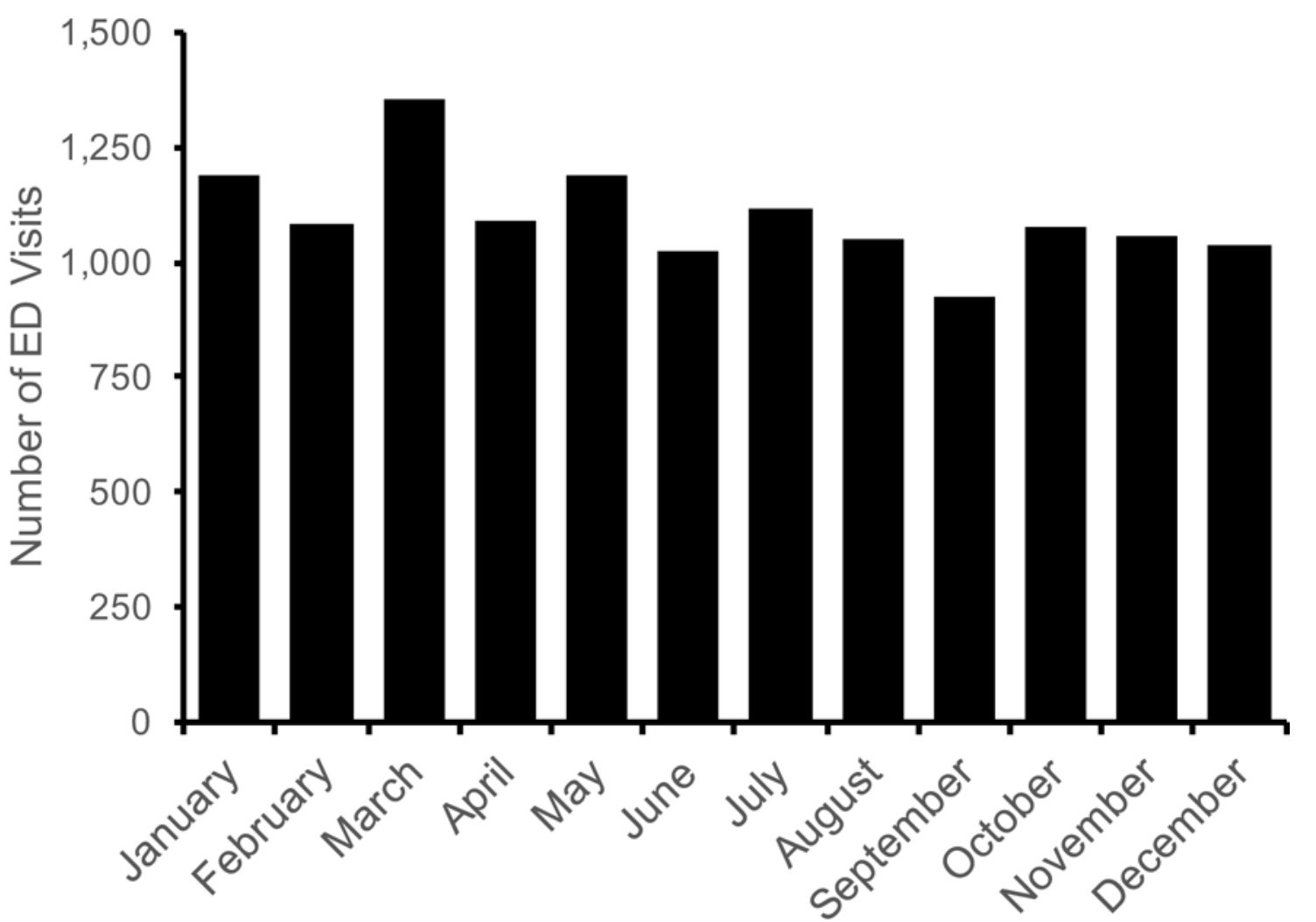

Figure 2.

Temporal trends in the volume of visits with a diagnosis of acute otitis media and associated complications in hospital-based emergency departments in the United States (2009 through 2011). 


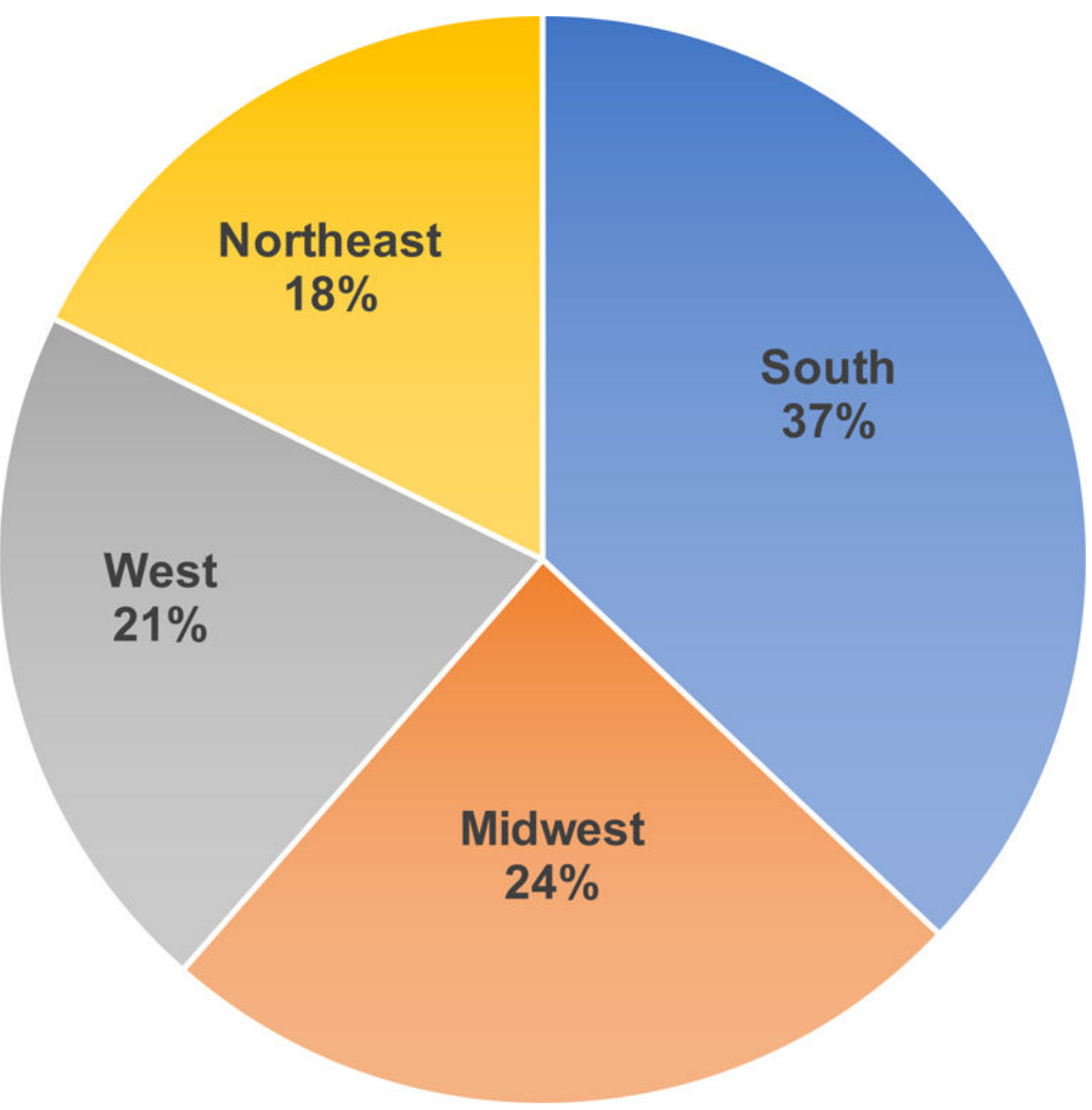

Figure 3.

Weighted estimates of incidence of complications associated with acute otitis media in visits to hospital-based emergency departments stratified by census-defined regions (2009 through 2011). 


\section{Table 1}

Types of Complications Associated with Diagnosis of Acute Otitis Media in Emergency Department Encounters (2009 Through 2011).

\begin{tabular}{ll}
\hline Complication Type & N (Weighted Frequency, \%) \\
\hline All visits & $5,811,127(100 \%)$ \\
No complication & $5,795,277(99.74 \%)$ \\
Complication ${ }^{*}$ & $15,243(0.26 \%)$ \\
Acute mastoiditis & $9,387(0.16 \%)$ \\
Labyrinthitis & $3,402(0.06 \%)$ \\
Facial paresis & $1,677(0.03 \%)$ \\
Subperiosteal abscess & $560(0.01 \%)$ \\
Gradenigo syndrome & $366(0.006 \%)$ \\
Meningitis & $130(0.002 \%)$ \\
Venous sinus thrombosis & $113(0.002 \%)$ \\
Petrositis & $97(0.002 \%)$ \\
Intracranial abscess & $92(0.002 \%)$ \\
Encephalocele & $14(0.0002 \%)$ \\
CSF Otorrhea & $12(0.0002 \%)$ \\
Labyrinthine fistula & $0(0 \%)$ \\
\hline
\end{tabular}




\section{Table 2}

Characteristics of Emergency Department Encounters for Diagnosis of Acute Otitis Media, Stratified by Development of Associated Complications (2009 Through 2011).

\begin{tabular}{|c|c|c|c|c|}
\hline \multirow[b]{2}{*}{ Variable } & \multicolumn{3}{|c|}{ Weighted Frequency, \% (SE) } & \multirow[b]{2}{*}{$P$ Value } \\
\hline & $\begin{array}{l}\text { All Patients, } \mathrm{N}= \\
5, \mathbf{8 1 1 , 1 2 7}\end{array}$ & Complications, $N=15,243$ & $\begin{array}{c}\text { No Complications, } \mathrm{N}= \\
\mathbf{5 , 7 9 5 , 8 8 4}\end{array}$ & \\
\hline Mean Age & $10.1(0.1)$ & $37.0(0.6)$ & $10.0(0.1)$ & $<.0001$ \\
\hline Age category & & & & $<.0001$ \\
\hline $0-18$ years & $79.9 \%(0.4)$ & $24.1 \%(1.3)$ & $80.1 \%(0.4)$ & \\
\hline$>18$ years & $20.1 \%(0.4)$ & $75.9 \%(1.3)$ & $19.9 \%(0.4)$ & \\
\hline Gender & & & & $<.0001$ \\
\hline Male & $50.1 \%(0.1)$ & $44.6 \%(0.1)$ & $50.1 \%(0.1)$ & \\
\hline Female & $49.9 \%(0.1)$ & $55.4 \%(1.1)$ & $49.9 \%(0.1)$ & \\
\hline Weekend visit, Saturday or Sunday & $34.3 \%(0.1)$ & $29.7 \%(0.8)$ & $34.3 \%(0.1)$ & $<.0001$ \\
\hline Median household income & & & & $<.0001$ \\
\hline$\$ 1-\$ 38,999$ & $35.4 \%(0.9)$ & $31.2 \%(1.1)$ & $35.4 \%(0.9)$ & \\
\hline$\$ 39,000-\$ 47,999$ & $30.9 \%(0.7)$ & $28.1 \%(1.0)$ & $30.9 \%(0.7)$ & \\
\hline$\$ 48,000-\$ 63,999$ & $21.6 \%(0.6)$ & $24.2 \%(1.0)$ & $21.6 \%(0.6)$ & \\
\hline$\$ 64,000$ or more & $12.0 \%(0.5)$ & $16.5 \%(0.9)$ & $12.0 \%(0.5)$ & \\
\hline Primary insurer & & & & $<.0001$ \\
\hline Medicaid & $54.7 \%(0.6)$ & $29.4 \%(1.1)$ & $54.8 \%(0.6)$ & \\
\hline Private insurance & $27.5 \%(0.6)$ & $31.7 \%(0.9)$ & $27.5 \%(0.6)$ & \\
\hline Self-pay & $11.6 \%(0.2)$ & $16.0 \%(0.8)$ & $11.6 \%(0.2)$ & \\
\hline Other & $3.6 \%(0.2)$ & $4.0 \%(0.4)$ & $3.6 \%(0.2)$ & \\
\hline Medicare & $2.2 \%(0.1)$ & $18.0 \%(0.9)$ & $2.1 \%(0.1)$ & \\
\hline No charge & $0.3 \%(0.05)$ & $0.9 \%(0.2)$ & $0.3 \%(0.05)$ & \\
\hline Hospital region & & & & .0403 \\
\hline South & $40.4 \%(1.1)$ & $37.0 \%(1.4)$ & $40.4 \%(1.1)$ & \\
\hline Midwest & $25.9 \%(1.0)$ & $24.4 \%(1.3)$ & $25.9 \%(1.1)$ & \\
\hline West & $16.9 \%(0.8)$ & $20.8 \%(1.2)$ & $16.9 \%(0.8)$ & \\
\hline Northeast & $16.8 \%(0.8)$ & $17.7 \%(1.0)$ & $16.8 \%(0.8)$ & \\
\hline Hospital trauma designation & & & & .0403 \\
\hline Yes & $24.2 \%(1.2)$ & $26.8 \%(1.3)$ & $24.2 \%(1.2)$ & \\
\hline
\end{tabular}


Table 3

Multivariable logistic regression assessing predictors of complication.

\begin{tabular}{|c|c|c|c|}
\hline Variable & Odds Ratio (OR) & 95\% Confidence Interval & $P$ value \\
\hline Weekday (vs. weekend) & 1.18 & $1.08-1.28$ & 0.0002 \\
\hline \multicolumn{4}{|l|}{ Age Category } \\
\hline $18-44$ vs. $<18$ & 2.11 & $1.65-2.69$ & $<0.0001$ \\
\hline $45-64$ vs. $<18$ & 1.24 & $0.84-1.89$ & 0.2667 \\
\hline$>64$ vs. $<18$ & 0.81 & $0.46-1.43$ & 0.4591 \\
\hline Male (vs. Female) & 1.17 & $1.05-1.29$ & 0.0032 \\
\hline \multicolumn{4}{|l|}{ Hospital region } \\
\hline Northeast vs. West & 0.65 & $0.54-0.78$ & 0.0038 \\
\hline Midwest vs. West & 0.71 & $0.59-0.78$ & 0.1561 \\
\hline South vs. West & 0.76 & $0.64-0.90$ & 0.8406 \\
\hline \multicolumn{4}{|l|}{ Median household income quartile } \\
\hline$<\$ 39,000$ vs. $>\$ 64,000$ & 0.78 & $0.66-0.93$ & 0.0006 \\
\hline$\$ 39,000-\$ 47,999$ vs. $>\$ 64,000$ & 0.89 & $0.76-1.04$ & 0.4115 \\
\hline$\$ 48,000-\$ 63,999$ vs. $>\$ 64,000$ & 1.03 & $0.89-1.19$ & 0.0094 \\
\hline Trauma (vs. non-trauma) hospital & 1.48 & $1.26-1.74$ & $<0.0001$ \\
\hline \multicolumn{4}{|l|}{ Teaching status } \\
\hline Metropolitan teaching (vs. non-metropolitan) & 1.82 & $1.39-2.39$ & $<0.0001$ \\
\hline \multicolumn{4}{|l|}{ Primary payer } \\
\hline Medicaid (vs. Medicare) & 0.81 & $0.67-0.98$ & 0.0274 \\
\hline Private insurance (vs. Medicare) & 0.92 & $0.79-1.07$ & 0.2868 \\
\hline Self-pay (vs. Medicare) & 0.72 & $0.60-0.86$ & 0.0002 \\
\hline No charge (vs. Medicare) & 1.16 & $0.67-1.17$ & 0.5942 \\
\hline Other (vs. Medicare) & 0.90 & $0.70-1.65$ & 0.4317 \\
\hline
\end{tabular}




\section{Table 4}

Outcome of Emergency Department Encounters for Diagnosis of Acute Otitis Media, Stratified by Development of Complications (2009 Through 2011).

\begin{tabular}{|c|c|c|c|c|}
\hline \multirow[b]{2}{*}{ Variable } & \multicolumn{3}{|c|}{ Weighted Frequency, \% (SE) } & \multirow[b]{2}{*}{$P$ Value } \\
\hline & All Patients, $N=5,811,127$ & Complications, $N=15,243$ & No Complications, $N=\mathbf{5 , 7 9 5 , 8 8 4}$ & \\
\hline Outcome & & & & $<.0001$ \\
\hline Admitted & $0.56 \%(0.06)$ & $43.6 \%(1.3)$ & $0.4 \%(0.1)$ & \\
\hline Treated and released & $99.44 \%(0.06)$ & $56.4 \%(1.3)$ & $99.6 \%(0.1)$ & \\
\hline \multicolumn{5}{|l|}{ Diagnostic test used } \\
\hline CT head & $0.06 \%(0.01)$ & $2.3 \%(0.4)$ & $0.1 \%(0.01)$ & $<.0001$ \\
\hline MRI head & $0.001 \%(0.0003)$ & $0.17 \%(0.08)$ & $0.0007 \%(0.0002)$ & 0.0311 \\
\hline \multicolumn{5}{|l|}{ Procedure performed } \\
\hline Myringotomy and tube & $0.01 \%(0.002)$ & $0.24 \%(0.12)$ & $0.01(0.001)$ & 0.0143 \\
\hline
\end{tabular}

